\title{
BMJ Open Women's prepregnancy lipid levels and number of children: a Norwegian prospective population-based cohort study
}

\author{
Aleksandra Pirnat, ${ }^{1}$ Lisa A DeRoo, ${ }^{1}$ Rolv Skjærven, ${ }^{1,2}$ Nils-Halvdan Morken ${ }^{3,4}$
}

To cite: Pirnat A, DeRoo LA, Skjærven R, et al. Women's prepregnancy lipid levels and number of children: a Norwegian prospective population-based cohort study. BMJ Open 2018;8:e21188. doi:10.1136 bmjopen-2017-021188

- Prepublication history for this paper is available online. To view these files, please visit the journal online (http://dx.doi org/10.1136/bmjopen-2017021188).

Received 19 December 2017 Revised 14 March 2018 Accepted 16 May 2018

\section{Check for updates}

${ }^{1}$ Department of Global Public Health and Primary Care, University of Bergen, Bergen, Norway

${ }^{2}$ (The Medical Birth Registry of Norway), Norwegian Institute of Public Health, Bergen, Norway ${ }^{3}$ Department of Clinical Science, University of Bergen, Bergen, Norway

${ }^{4}$ Department of Obstetrics and Gynecology, Haukeland University Hospital, Bergen, Norway

Correspondence to Aleksandra Pirnat; pirnatdraleksandra@gmail.com

\section{ABSTRACT}

Objective To study prepregnancy serum lipid levels and the association with the number of children.

Design Prospective, population-based cohort.

Setting Linked data from the Cohort of Norway and the Medical Birth Registry of Norway.

Participants 2645 women giving birth to their first child during 1994-2003 (488 one-child mothers and 2157 women with $\geq 2$ births) and 1677 nulliparous women. Main outcome measures ORs for no and one lifetime pregnancy (relative to $\geq 2$ pregnancies) obtained by multinomial logistic regression, adjusted for age at examination, education, body mass index (BMI), smoking, time since last meal and oral contraceptive use.

Results Assessed in quintiles, higher prepregnant triglyceride (TG) and TG to high-density lipoprotein (TG:HDL-c) ratio levels were associated with increased risk of one lifetime pregnancy compared with having $\geq 2$ children. Compared with the highest quintile, women in the lowest quintile of HDL cholesterol levels had an increased risk of one lifetime pregnancy (OR 1.7, 95\% $\mathrm{Cl} 1.2$ to 2.4 ), as were women with the highest low-density lipoprotein (LDL) cholesterol, TG and TG:HDL-c ratio quintiles (compared with the lowest) (OR 1.2, 95\% $\mathrm{Cl} 0.8$ to 1.7; OR 2.2, $95 \% \mathrm{Cl} 1.5$ to 3.2; and $\mathrm{OR} 2.2,95 \% \mathrm{Cl} 1.5$ to 3.2 , respectively). Similar effects were found in women with $\mathrm{BMI} \geq 25$ and the highest LDL and total cholesterol levels in risk of lifetime nulliparity.

Conclusion Women with unfavourable prepregnant lipid profile had higher risk of having no or only one child. These findings substantiate an association between prepregnant serum lipid levels and number of children. Previously observed associations between low parity and increased cardiovascular mortality may in part be due to pre-existing cardiovascular disease lipid risk factors.

\section{INTRODUCTION}

Cardiovascular disease (CVD) is an important public health problem and remains the number one cause of death in women. ${ }^{1}$ Reproductive history is important in evaluating health risks in women, as pregnancy may unmask a woman's predisposition for CVD. ${ }^{1}$ Several studies have reported increased CVD mortality among women with no or only
Strengths and limitations of this study

- This is a large population-based study with data collected before pregnancy.

- Linkage with the Medical Birth Registry of Norway provided complete registration of total reproduction.

- Limitations include lack of data on family planning, dietary intake, duration of oral contraceptive use, APOE genotype, low-grade inflammation and thyroid status.

- Non-fasting lipid measurements were used; however, adjustments in our analyses for time since last meal did not change the results.

one lifetime pregnancy. ${ }^{2-4}$ Efforts to elucidate the association between the number of children and the risk of female CVD have been inconclusive. ${ }^{13}$ Proposed explanations are lifestyle risk factors associated with childrearing, ${ }^{5}$ sex hormone fluctuations, protective effect of future pregnancies, ${ }^{3}$ lifestyle factors prior to conception such as elevated blood pressure and obesity, ${ }^{6}$ as well as metabolic irregularities triggered by gestation. ${ }^{1}$ Detection of high-density lipoprotein (HDL) cholesterol and apolipoprotein B (ApoB) in follicular fluid from oocytes ${ }^{78}$ suggests a relation between lipids and female reproductive function. More recent studies have reported associations between lipids and fertility in both sexes. ${ }^{9}$ Low parity (as a feature of subfecundity) and cardiovascular events may share common pathophysiological mechanisms. ${ }^{10}$

While the role of serum lipids in cardiometabolic health is well established, showing low HDL and high triglycerides (TGs) to be strong predictors of $\mathrm{CVD},{ }^{11}$ their role in reproduction is uncertain. It is also uncertain whether women with no or one lifetime pregnancy have a higher CVD risk to begin with, or whether future pregnancies may reduce the GVD risk. 
We pursued this question by exploring the extent to which prepregnant serum lipid levels of total, HDL and low-density lipoprotein (LDL) cholesterol, TG and TG:HDL-c ratio are associated with having no and one lifetime pregnancy.

\section{MATERIALS AND METHODS}

\section{Study design and population}

We used linked data from the Cohort of Norway (CONOR) and the Medical Birth Registry of Norway (MBRN). CONOR is a population-based collection of health data and blood samples provided by participants older than 20 years of age residing in several different regions in Norway during $1994-2003 .{ }^{12}$ Our subset included women with no children at the time of examination with standardised measurements of height, weight and non-fasting lipids levels. Lifestyle factors were obtained through an extensive questionnaire that collected self-reported information on smoking, oral contraceptive (OC) use, self-reported status on receipt of social security benefits, attained level of education and various lifestyle factors. ${ }^{12}$ Education in Norway consists of primary school (7 years), lower secondary school (3 years), upper secondary school (3 years) and higher education. The first 10 years are obligatory.

The MBRN has since 1967 recorded data on all deliveries in the country after 16 th week of gestation. ${ }^{13}$ Based on mandatory notification, midwives and doctors report information using standard forms throughout pregnancy and at the time of delivery. The registry includes demographic information, mother's health prior and during pregnancy, complications in pregnancy and perinatal outcome. Using the unique national identification number given to all Norwegian citizens, each woman was linked to all her subsequent births (if any) after participating in CONOR. Women reporting no children in CONOR at the time of examination and with no valid records in the MBRN were considered having no pregnancies.

Women with baseline assessment of lifestyle factors in CONOR were linked to the MBRN. We defined one-child mothers as women being 6 years out from their first pregnancy and with no additional births registered in the MBRN.

\section{Preconception measurements}

Non-fasting blood samples were analysed on a Hitachi 911 Auto Analyzer (Hitachi, Mito, Japan). ${ }^{12}$ Applied reagents were from Boehringer Mannheim (Mannheim, Germany). Serum concentrations of total cholesterol, HDL cholesterol and TG were analysed subsequent to sampling. The total cholesterol, HDL cholesterol levels and TGs were measured by an enzymatic method. The day-to-day coefficients of variation were $2.4 \%$ and $0.7 \%-$ $1.3 \%$ for total cholesterol, HDL cholesterol and TG, respectively. To calculate LDL, we used the Friedewald formula ${ }^{14}$ : total serum cholesterol minus HDL cholesterol minus one-fifth of the TG concentration. LDL cholesterol levels were calculated only for participants with TG concentrations below $4.5 \mathrm{mmol} / \mathrm{L} .{ }^{6}{ }^{14}$ Accordingly, the TG:HDL-c ratio was expressed as $\mathrm{mmol} / \mathrm{L}$.

Trained personnel measured the height and weight, with the participants wearing light clothes and no shoes; measurements were taken as follows: height to the nearest $1.0 \mathrm{~cm}$ and weight to the nearest $0.5 \mathrm{~kg}$. Body mass index (BMI) was calculated as weight in kilogram/(height in metres) $)^{2}$.

\section{Patients and public involvement}

Patients or the public were not directly involved in this study. The detailed explanation of the recruitment process and the obtainment of written informed consent for CONOR were provided elsewhere. ${ }^{12}$

\section{Statistical analyses}

The characteristics of the analysed women were presented as means with SD for continuous data and as number with percentages for categorical data. Differences between nulliparous women, one-child mothers and mothers with two or more children, as well as prepregnant health status, were analysed by $\chi^{2}$ tests and t-tests where appropriate. Linear associations across prepregnant lipid levels (in quintiles) for no and one lifetime pregnancy were assessed by $p$ values for trend. ORs of no and one lifetime pregnancy by lipid levels and TG:HDL-c ratio, when compared with women with two or more pregnancies, were calculated using multinomial logistic regression and adjusted for mother's age at examination, level of education (categorised in $<11$ years and $>11$ years of education), smoking (current smoker: yes, no), time since last meal, OC use (now, previously, never) and BMI (linear term). To extend each woman's likelihood of completing her birth record, we separately examined women who were 7 years out from their first pregnancy. About $95 \%$ of Norwegian women will complete their second pregnancy within 7 years. ${ }^{4}$ To test the effect of (prepregnant) BMI, we stratified the main analyses by BMI $(<25$ and $\geq 25)$. To avoid influence from age at first delivery on the number of children, we excluded women older than 34 years at the time of first delivery in a subanalysis. Additionally, we performed sensitivity analyses including only mothers who were 22-30 years old at the time of first delivery. Using presence of a partner (ever) as a proxy for exposure to pregnancy among nulliparous women, we performed logistic regression in a subanalysis (nulliparous vs women with $\geq 2$ births) including only women with a reported partner (ever). All analyses were performed using the Statistical Package for Social Sciences (SPSS V.22.0 and V.23.0).

\section{RESULTS}

There were 4743 women with baseline assessment of lifestyle factors in CONOR (1994-2003) that were linked to the MBRN. We excluded 421 women with pregnancy 


\section{Exclusions}

\section{3 women \\ Preconception examination in CONOR \\ (1994-2003) and linked to the MBRN}

421 women
-Pregnant at examination (139)
-Unsure pregnancy status (157),
-Missing lipid assessment (125)

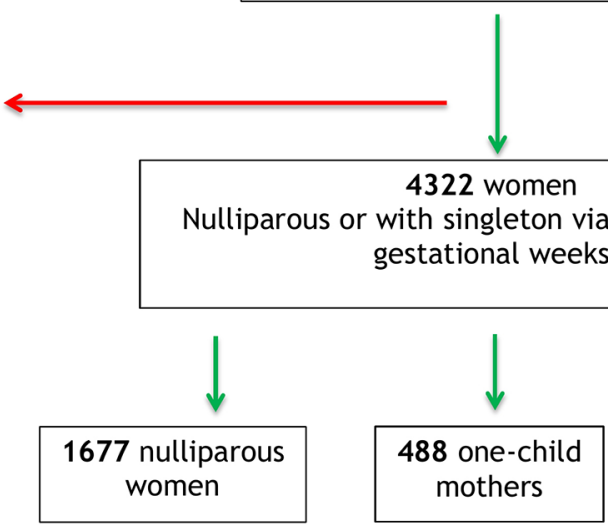

able births $\geq 22$

with singleton viat

gestational weeks

Figure 1 Norwegian women examined in the Cohort of Norway (CONOR) before conception of their first pregnancy and with linked data from the Medical Birth Registry of Norway (MBRN).

at the time of examination ( $\mathrm{n}=139)$, unsure pregnancy status $(n=157)$ and missing lipid assessments $(n=125)$. Thus, 4322 women were included in the analyses (1677 nulliparous, 488 one-child mothers and 2157 women with $\geq 2$ births; see figure 1 ). Subanalyses included only women with reported partners (228 nulliparous and 216 mothers with $\geq 2$ births).

The characteristics of the included women are given in table 1. Nulliparous women were older at the time of examination, had higher BMI and were more frequent smokers compared with women with two or more births. A higher proportion of nulliparous women had $>11$ years of education. One-child mothers had higher mean age both at examination and at delivery (29.5 vs 26.7 and 32.3 vs 29.9 , respectively), were more often smokers and had lower education than mothers with $\geq 2$ births. The mean BMI prior to pregnancy was higher in one-child mothers (24.2 vs 23.5), whereas the mean years from examination to first delivery were similar for the two groups. Women with no and one child were less frequent users of OCs at the time of examination compared with mothers with $\geq 2$ births $(27.4 \%, 34.6 \%$ and $48.9 \%$, respectively).

The proportion of diabetes at first delivery in one-child mothers was higher than in women with two or more

Table 1 Characteristics of 4322 Norwegian women in the Cohort of Norway, 1994-2003, with no, 1 or $\geq 2$ children

\begin{tabular}{|c|c|c|c|}
\hline Mean values & 1677 no child & 488 one child & $2157 \geq 2$ children \\
\hline Age (SD) at examination & $30.5(2.1)$ & $29.5(5.2)$ & $26.7(4.0)$ \\
\hline Age (SD) at first delivery & - & $32.3(4.9)$ & $29.9(3.8)$ \\
\hline Years (SD) from examination to first pregnancy & - & $3.7(2.1)$ & $4.1(2.3)$ \\
\hline BMI (SD) at examination* & $24.8(5.1)$ & $24.2(4.5)$ & $23.5(3.4)$ \\
\hline \multicolumn{4}{|l|}{ OC use* } \\
\hline Now & $455(27.4)$ & $168(34.6)$ & $1047(48.9)$ \\
\hline Previously & $724(43.5)$ & 239 (49.2) & $779(36.4)$ \\
\hline Never & $484(29.1)$ & 79 (16.3) & $317(14.8)$ \\
\hline \multicolumn{4}{|l|}{ Smoking at examination* } \\
\hline Yes & 537 (32.2) & $182(37.4)$ & $462(21.5)$ \\
\hline No & $1132(67.8)$ & $304(62.6)$ & $1685(78.5)$ \\
\hline \multicolumn{4}{|l|}{ Education* } \\
\hline$<11$ years & $312(18.8)$ & $127(26.3)$ & $300(14.1)$ \\
\hline$\geq 11$ years & $1344(81.2)$ & 356 (73.7) & $1834(85.9)$ \\
\hline
\end{tabular}

Values are numbers (percentages) unless stated otherwise.

*Missing data on smoking: 8 nulliparous, 2 one-child mothers and 10 women with $\geq 2$ children; BMI, 10 nulliparous; education, 21 nulliparous, 5 one-child mothers and 23 women with $\geq 2$ children; OC use: 4 nulliparous, 2 one-child mothers and 14 women with $\geq 2$ children.

$\mathrm{BMI}$, body mass index; OC, oral contraceptive. 
Table 2 ORs with $95 \% \mathrm{Cl}$ for no and one lifetime pregnancy (reference: women with $\geq 2$ pregnancies) by prepregnant lipid quintiles in 4322 women in the Cohort of Norway, 1994-2003

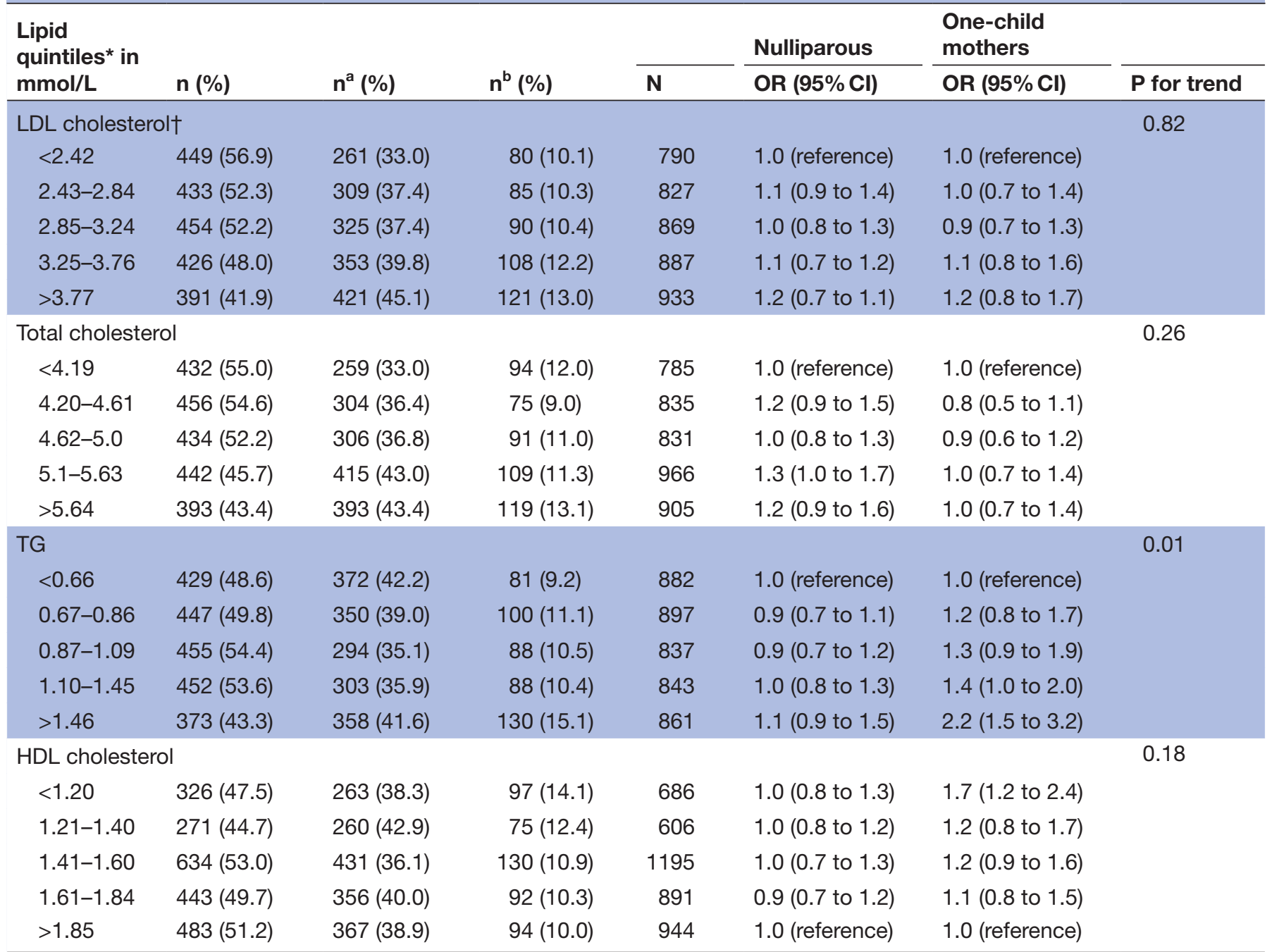

The estimates were obtained by multinomial logistic regression and adjusted for age at examination, educational level, smoking, time since last meal, oral contraceptive use and body mass index (linear term).

Number of women: with $\geq 2$ children ( $\mathrm{n}$, reference group), nulliparous women $\left(\mathrm{n}^{\mathrm{a}}\right)$, one-child mothers $\left(\mathrm{n}^{\mathrm{b}}\right)$ and total number of women within the category $(\mathrm{N})$.

${ }^{*}$ Quintiles calculated on a total sample prior to pregnancy.

†Missing data within lipids on 16 cases of LDL and 2 cases of TG.

HDL, high-density lipoprotein; LDL, low-density lipoprotein; TG, triglycerides.

births $(1.4 \%$ vs $0.9 \%, \mathrm{p}=0.30)$. Polycystic ovary syndrome (PCOS) was rare and we only had three cases in our material. A significantly higher number of one-child mothers had in vitro fertilisation (IVF) in their first pregnancy ( $7.2 \%$ vs $2.6 \%$ in women with $\geq 2$ births; $p<0.001$ ) (data not shown). This latter finding remained after excluding mothers older than 34 years at first delivery.

ORs with $95 \%$ CIs for no and one lifetime pregnancy (vs $\geq 2$ lifetime pregnancies) by lipid levels (in quintiles) are presented in table 2 and figure 2. Significant trends in ORs for one lifetime pregnancy across TG and TG:HDL-c ratio quintiles were observed ( $p$ trend $=0.01$ ). The OR for having one lifetime pregnancy for women with the highest TG quintile compared with the lowest quintile was 2.2 (95\% CI 1.5 to 3.2). The ORs for having one lifetime pregnancy for women with TG:HDL-c ratio levels in the two highest quintiles were 1.7 (95\% CI 1.2 to 2.5 ) and 2.2 (95\% CI 1.5 to 3.2), respectively, compared with the lowest quintile. There were no significant trends for LDL cholesterol, total cholesterol or HDL cholesterol, although the ORs of one lifetime pregnancy for the lowest HDL quintile were 1.7 (95\% CI 1.2 to 2.4) and for the highest LDL quintile 1.2 (95\% CI 0.8 to 1.7). We found no increased risk of being nulliparous by serum lipid levels except for the highest LDL and total cholesterol levels, and these estimates were not persuasive (OR 1.2 (95\% CI 0.9 to 1.6 ) and 1.2 (95\% CI 0.9 to 1.5), respectively). Truncation of data to extend the time for each woman to complete her birth record (to 7 years) did not appreciably alter the results, neither did 
TG/HDL-Ratio in nulliparous women
TG/HDL-Ratio

in one-child mothers
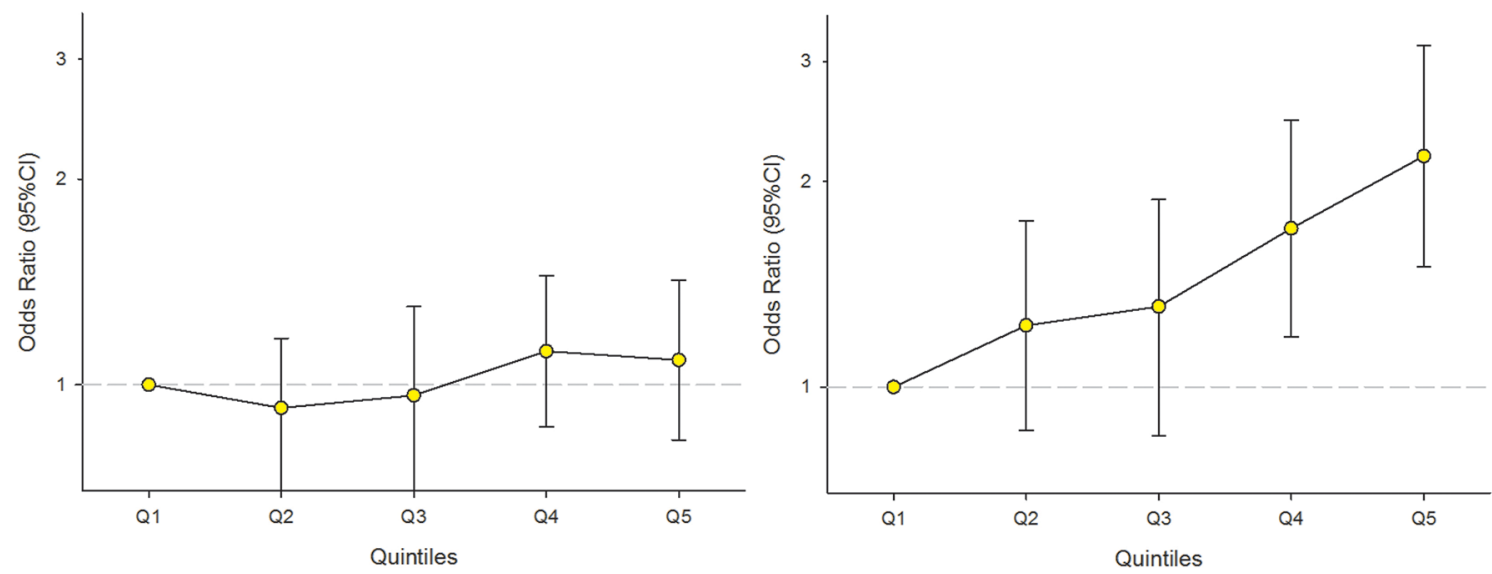

Figure 2 ORs with 95\% Cl for no and one lifetime pregnancy (reference: women with $\geq 2$ pregnancies) by TG:HDL-c ratio quintiles in 4322 women in the Cohort of Norway, 1994-2003. The estimates were obtained by multinomial logistic regression and adjusted for age at examination, educational level, smoking, time since last meal, oral contraceptive use and body mass index (linear term). TG:HDL-c, triglycerides to high-density lipoprotein ratio.

exclusion of women older than 34 years at the time of first delivery nor the additional restriction of our analyses to mothers aged 22-30 years at first pregnancy. The similar effects of prepregnant lipids as in one-child mothers were observed when subanalyses (nulliparous vs $\geq 2$ births) were performed on women who had a partner (as a proxy for ever being exposed to pregnancy). For women with partner, the risk of having no children was increased among the women in the highest quintiles of TG and TG:HDL-c ratio (compared with the lowest quintiles) and also for those in the lowest HDL quintile (compared with the highest) (OR 1.9, 95\% CI 0.9 to 4.2 ; OR $2.0,95 \%$ CI 1.0 to 4.1 ; and OR $1.6,95 \%$ CI 0.7 to 3.6 , respectively).

Stratified analyses by BMI are presented in table 3 . In women with $\mathrm{BMI} \geq 25$ there were significant trends in ORs of having no children or one child across increasing levels of prepregnant total cholesterol, TG and TG:HDL-c ratio quintiles ( $\mathrm{p}$ trend $=0.04$ and $<0.001$, respectively). The adjusted ORs of one lifetime pregnancy for women with $\mathrm{BMI} \geq 25$ and TG levels in the two highest quintiles were 2.1 (95\% CI 0.9 to 4.8 ) and 3.5 (95\% CI 1.6 to 7.4 ), and for the two highest TG:HDL-c ratio quintiles 3.1 (95\% CI 1.3 to 7.4 ) and 4.3 (95\% CI 1.9 to 10.0 ) compared with women in the lowest respective quintile. The risk of one lifetime pregnancy was also significantly increased for women with $\mathrm{BMI} \geq 25$ and the highest LDL and total cholesterol, as well as the lowest HDL quintiles (OR 1.8 (95\% CI 0.8 to 3.8$), 1.2$ (95\% CI 0.6 to 2.4$)$ and $2.6(95 \%$ CI 1.3 to 5.3), respectively). Similarly, the ORs of having no pregnancy (in women with BMI $\geq 25)$ were 1.7 (95\% CI 1.0 to 3.0$), 2.8$ (95\% CI 1.7 to 4.7$)$ and 3.6 (95\% CI 2.1 to 6.1) for women with the highest LDL, TG and TG:HDL-c ratio quintiles, respectively, compared with women with the lowest quintile. An increased risk of having no children was also found for the overweight and obese women with the lowest HDL quintile (OR 1.9, 95\% CI 1.2 to 3.0). Unlike in one-child mothers, the risk of having no pregnancy among overweight and obese women with higher total cholesterol levels only slightly changed from the main results. In women with prepregnant $\mathrm{BMI}<25$, there were significant trends in the risk of having one lifetime pregnancy across increasing levels of prepregnant TG $(p$ trend=0.04), TG:HDL-c ratio ( $p$ trend=0.04) and HDL quintiles $(p$ trend $=0.05)$. There were increased risks of one lifetime pregnancy in the highest TG quintile (OR $1.9,95 \%$ CI 1.2 to 3.0 ) and the two highest TG:HDL-c ratio quintiles (OR 1.6, 95\% CI 1.0 to 2.4; and OR 1.8, $95 \%$ CI 1.2 to 2.8 , respectively), as well as the lowest HDL quintile (OR 1.7, 95\% CI 1.1 to 2.6). The risks of no and one lifetime pregnancy with higher LDL and total cholesterol levels only slightly changed compared with our main results.

\section{DISCUSSION}

Prepregnant lipid levels were associated with having one lifetime pregnancy. Women with high levels of LDL, TG and TG:HDL-c ratio, as well as low HDL levels, measured years before conception, were at increased risk of having only one lifetime pregnancy. High levels of LDL and total cholesterol were associated with having no children, while in overweight and obese women this was true for all the lipids examined.

These findings provide a possible biological underpinning for a joint mechanistic pathway for reduced fertility and cardiovascular conditions. ${ }^{10}$ Our study suggests that the previously observed association between low parity and increased CVD risk may be confounded by pre-existing adverse lipid levels. This does not support the hypothesis that having additional pregnancies reduces 


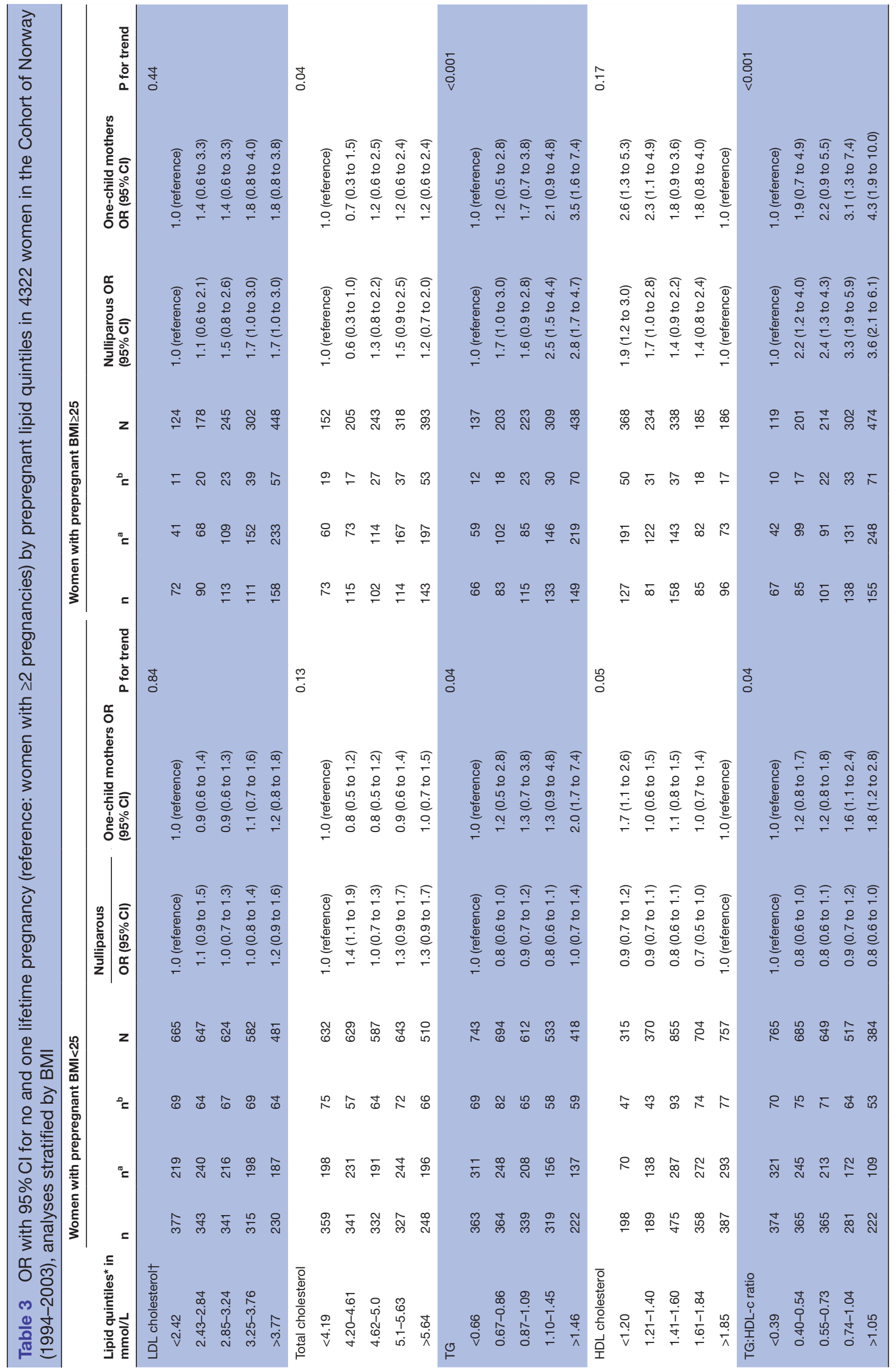


CVD risk. ${ }^{3}$ Rather, unfavourable lipid profiles may be related to both subfertility and later CVD.

There is a lack of studies evaluating the relation between preconception lipid levels and fertility in women. The LIFE (Longitudinal Investigation of Fertility and the Environment) study found concentrations of free cholesterol to be associated with fecundity in both sexes. ${ }^{9}$ In contrast to our study, TGs and total cholesterol were not found to be significant in individual and couple-based adjusted models (as well as two other measured lipid components: phospholipids and total lipids); however, the authors used a different study design and lipid measurement methods. In accordance with our findings is the Framingham Heart Study, which detected a trend towards TG elevation and lower HDL serum levels among women with self-reported infertility (as not achieving pregnancy for $\geq 1$ year). ${ }^{15}$ The presence of HDL cholesterol and ApoB in follicular fluid from human oocytes suggests that these lipids play a direct role in reproduction. ${ }^{7816}$ Previous animal studies have reported an association between dyslipidaemia and infertility ${ }^{17}$ Posed explanations have been that abnormalities in HDL metabolism including change in structure, concentration or function compromise female fertility. ${ }^{7816}$ It has been suggested that genetic polymorphisms that alter function in proteins engaged in cholesterol metabolism may affect human fertility. ${ }^{18} 19$ One of the possible molecular mechanisms could be through a mediating role of HDL on paraoxonase 1 activity. Paraoxonase is an HDL-associated enzyme that inhibits LDL oxidation, and thus protects cells from oxidative stress. ${ }^{20}$ Its stability and binding affinity are strongly influenced by changes in shape and size of HDL particles. ${ }^{21}$ These changes may lead to decreased antioxidative capacity and consecutively oxidative stress. Oxidative stress is associated with adverse cardiovascular and fertility outcomes, including atherosclerosis, PCOS, pre-eclampsia, endometriosis and infertility. ${ }^{1922}$ A recent study in women of reproductive age with upper normal ranges of thyroid-stimulating hormone has suggested a direct link between unfavourable lipid profile and increased oxidative membrane damage. ${ }^{23}$

Recent insights suggest TG:HDL-c ratio to be a reliable marker of insulin resistance and atherogenicity, ${ }^{24}$ highlighting its ability to identify insulin resistance in apparently healthy individuals. ${ }^{25}$ Observed higher levels of TG:HDL-c ratio in our study are indicative of possible pre-existing metabolic risk factors among women with one lifetime pregnancy, as well as a subpopulation of nulliparous women (overweight, obese and with reported partners-as a proxy for exposed to pregnancy). This is also consistent with increasing rates of infertility in both sexes among population with metabolic syndrome. ${ }^{9}$ The higher proportion of diabetes in this group of women further supports this notion. In agreement, the Japan Nurses' Health Study reported a significant increase in the risk of diabetes in young nulliparous women $(<45$ years of age) with ovarian infertility. ${ }^{26}$ Accordingly, the Framingham Heart Study found infertile premenopausal women to have increased odds of diabetes and obesity. ${ }^{15}$
Given the accompanying metabolic irregularities among major causes of female infertility, ${ }^{152}$ substantially higher proportion of IVF treatment among one-child mothers indirectly supports metabolic implications. The latter finding remains after exclusion of women older than 34 years at the time of first delivery.

Dyslipidaemia is associated with PCOS. ${ }^{28-30}$ However, we only identified three women with PCOS in our study sample. Thus, the presence of subclinical forms or under-reporting may be present.

In accordance with the literature,${ }^{27}{ }^{31}$ the risk of having no and only one child showed strong effects in overweight and obese women (BMI $\geq 25$ ) in stratified analyses (table 3). Nevertheless, the higher risk of having only one child remained in normal-weight women $(\mathrm{BMI}<25)$ with the lowest HDL quintile and the highest TG and TG:HDL-c ratio quintiles. These findings mirror observations from the literature of metabolic irregularities among normal-weight women as an independent risk factor for future fertility impairments. ${ }^{32} 33$ The LIFE study reported both female and male lipid concentrations to affect fecundity, irrespective of their BMI. ${ }^{9}$

Compared with women with two or more pregnancies, total cholesterol levels above clinically recommended range were associated with risk of having no children, irrespective of BMI. The LIFE study reported a higher percentage of women with a history of irregular menstrual cycles in the highest quartile of free cholesterol. ${ }^{9}$ The Japan Nurses' Health Study found women with ovarian infertility to be at high risk of hypercholesterolaemia. ${ }^{26}$ In our study, total cholesterol levels were not associated with the risk of having one lifetime pregnancy, except among overweight and obese women. This could suggest that total cholesterol levels play varied roles in different subfecundity or infertility subtypes. In addition, nulliparous women in our study were older at examination and had higher BMI. Both age and obesity are associated with systemic oxidative stress. ${ }^{19}{ }^{22}$ It is possible that in such physiological environment, clinically abnormal levels of certain lipids might activate additional pathological processes that adversely affect reproductive function. ${ }^{28}$

In our study, women with one lifetime pregnancy had poorer lifestyle factors (BMI, smoking), were older and less educated. The lower mean education among one-child mothers is in agreement with a Nordic demographic study, ${ }^{34}$ which shows that later onset of childbearing is related to a lower number of children finally born in women with low education. Given that educational level and occupation are key indicators of socioeconomic status, ${ }^{35}$ observed lower parity among women with low education could also reflect unfavourable socioeconomic position as a limiting factor to further pregnancies. However, a study exploring age at first birth, parity and postreproductive mortality suggests that late childbearing in itself may be a signal of pre-existing poor health of a woman. ${ }^{36}$

The observed risk differences between nulliparous women and one-child mothers in our main results 
(figure 2, table 2) could be explained by the heterogeneity of causes for childlessness among nulliparous women in this cohort. The risk may, therefore, be diluted by low-risk groups of women who are voluntarily childless $^{37}$ or have not been exposed to pregnancy (ever). Given the lack of information on women's reproductive planning in our data, we tried to address this in a subanalysis including only women with reported partner (ever) as a proxy for being exposed to pregnancy. Here we found that the results for nulliparous women were similar to our main results for one-child mothers. Women with reported partner had higher risk of having no children (compared with partnered women with $\geq 2$ births) if their TG and TG:HDL-c ratio levels were in the highest quintiles and HDL in the lowest quintile (OR 1.9, 95\% CI 0.9 to 4.2; OR 2.0, 95\% CI 1.0 to 4.1 ; and OR $1.6,95 \%$ CI 0.7 to 3.6 , respectively). These findings support the role of serum lipids in lifetime nulliparity among women with partners.

\section{Strengths and limitations}

Our subset of women was from a large population-based health study with prepregnant health data. Linked data from the MBRN provided complete registration of total reproduction. The prospective design minimised the potential for bias. A weakness is that blood sampling was performed in a non-fasting state. Studies show that TG levels are sensitive to recent food intake, while cholesterol levels seem to be less affected. ${ }^{38}$ We addressed this by adjusting our analyses for time since last meal and the main results were unchanged, suggesting that non-fasting lipids are not likely to introduce a systematic bias. Non-fasting lipids have successfully been used in lipid and CVD research. ${ }^{9} 3940$

The study lacked data on apolipoprotein E genotype, CRP (C-reactive protein) and thyroid tests/thyroid antibodies, factors that all are found to affect female fertility. ${ }^{231}$ No assessments of duration or temporal proximity of OC use, dietary intake or stress were available; therefore, unmeasured confounding cannot be ruled out. Smoking adversely influences female fertility, ${ }^{42}$ with most of its effects attributed to HDL cholesterol decrease. ${ }^{43}$ We accounted for this in our analyses; however, smoking status of participants was only available at enrolment. The ethnic homogeneity of the included women may reduce the generalisability of our findings.

Unfavourable prepregnant lipid levels were associated with having no and one lifetime pregnancy. Women's metabolic homeostasis is important for reproduction and also has cardiometabolic implications. ${ }^{32} 44$ Pre-existing poor lipid and metabolic profiles could represent one of the possible linkages between previously observed reduced fertility and later CVD.

Acknowledgements We thank Allen Wilcox for his valuable comments on this paper.

Contributors All authors had full access to the data and are responsible for the integrity of the data. AP, RS, LAD and N-HM designed the study. AP and N-HM conducted the analyses. N-HM created the figure and AP created the tables and the flow chart. AP drafted the manuscript. N-HM, RS and LAD reviewed the preliminary analyses and initial draft and provided critical comments.
Funding This work was supported by the Norwegian Association for Public Health with a doctoral scholarship to AP. The Norwegian Association for Public Health had no role in the design and conduct of the study; in the collection, analysis and interpretation of the data; or in the preparation, review or approval of the manuscript.

Competing interests None declared.

Patient consent Not required.

Ethics approval The study was approved by the regional ethical review board REK-Vest (ref number 2013/118), and access to data was granted by the Steering Committee for CONOR and by the MBRN.

Provenance and peer review Not commissioned; externally peer reviewed.

Data sharing statement This data set contains personal data and cannot be made public due to confidentiality requirements according to Norwegian legislations. Researchers who are interested in analysing data from CONOR or the MBRN may apply to the appropriate organisations after having obtained all necessary approvals according to Norwegian legislations.

Open access This is an open access article distributed in accordance with the Creative Commons Attribution Non Commercial (CC BY-NC 4.0) license, which permits others to distribute, remix, adapt, build upon this work non-commercially, and license their derivative works on different terms, provided the original work is properly cited and the use is non-commercial. See: http://creativecommons.org/ licenses/by-nc/4.0/

(c) Article author(s) (or their employer(s) unless otherwise stated in the text of the article) 2018. All rights reserved. No commercial use is permitted unless otherwise expressly granted.

\section{REFERENCES}

1. Sanghavi M, Kulinski J, Ayers CR, et al. Association between number of live births and markers of subclinical atherosclerosis: The Dallas Heart Study. Eur J Prev Cardiol 2016;23:391-9.

2. Grundy E, Kravdal $\varnothing$. Reproductive history and mortality in late middle age among Norwegian men and women. Am J Epidemiol 2008;167:271-9.

3. Lv H, Wu H, Yin J, et al. Parity and Cardiovascular Disease Mortality: a Dose-Response Meta-Analysis of Cohort Studies. Sci Rep 2015;5:13411.

4. Skjaerven R, Wilcox AJ, Klungsøyr K, et al. Cardiovascular mortality after pre-eclampsia in one child mothers: prospective, population based cohort study. BMJ 2012;345:e7677.

5. Lawlor DA, Emberson JR, Ebrahim S, et al. Is the association between parity and coronary heart disease due to biological effects of pregnancy or adverse lifestyle risk factors associated with childrearing? Findings from the British Women's Heart and Health Study and the British Regional Heart Study. Circulation 2003:107:1260-4.

6. Magnussen EB, Vatten LJ, Lund-Nilsen TI, et al. Prepregnancy cardiovascular risk factors as predictors of pre-eclampsia: population based cohort study. BMJ 2007;335:978-81.

7. Gautier T, Becker S, Drouineaud V, et al. Human luteinized granulosa cells secrete apoB100-containing lipoproteins. J Lipid Res 2010;51:2245-52.

8. Von Wald T, Monisova Y, Hacker MR, et al. Age-related variations in follicular apolipoproteins may influence human oocyte maturation and fertility potential. Fertil Steril 2010;93:2354-61.

9. Schisterman EF, Mumford SL, Browne RW, et al. Lipid concentrations and couple fecundity: the LIFE study. J Clin Endocrinol Metab 2014;99:2786-94.

10. Parikh NI, Cnattingius S, Mittleman MA, et al. Subfertility and risk of later life maternal cardiovascular disease. Hum Reprod 2012;27:568-75.

11. Abdel-Maksoud MF, Eckel RH, Hamman RF, et al. Risk of coronary heart disease is associated with triglycerides and high-density lipoprotein cholesterol in women and non-high-density lipoprotein cholesterol in men. J Clin Lipidol 2012;6:374-81.

12. Naess O, Søgaard AJ, Arnesen E, et al. Cohort profile: cohort of Norway (CONOR). Int J Epidemiol 2008;37:481-5.

13. Irgens LM. The Medical Birth Registry of Norway. Epidemiological research and surveillance throughout 30 years. Acta Obstet Gynecol Scand 2000;79:435-9.

14. Friedewald WT, Levy RI, Fredrickson DS. Estimation of the concentration of low-density lipoprotein cholesterol in plasma, without use of the preparative ultracentrifuge. Clin Chem 1972;18:499-502. 
15. Mahalingaiah S, Sun F, Cheng JJ, et al. Cardiovascular risk factors among women with self-reported infertility. Fertil Res Pract 2017;3:7.

16. Miettinen HE, Rayburn $\mathrm{H}$, Krieger M. Abnormal lipoprotein metabolism and reversible female infertility in HDL receptor (SR-BI)deficient mice. J Clin Invest 2001;108:1717-22.

17. Lobaccaro JM, Gallot D, Lumbroso S, et al. Liver X Receptors and female reproduction: when cholesterol meets fertility!. J Endocrinol Invest 2013;36:55-60.

18. DeAngelis AM, Roy-O'Reilly M, Rodriguez A. Genetic alterations affecting cholesterol metabolism and human fertility. Biol Reprod 2014;91:117.

19. Gupta S, Ghulmiyyah J, Sharma R, et al. Power of proteomics in linking oxidative stress and female infertility. Biomed Res Int 2014;2014:1-26.

20. Lazaros L, Markoula S, Kyritsis A, et al. Paraoxonase gene polymorphisms and stroke severity. Eur J Neurol 2010;17:757-9.

21. Bhattacharyya T, Nicholls SJ, Topol EJ, et al. Relationship of paraoxonase 1 (PON1) gene polymorphisms and functional activity with systemic oxidative stress and cardiovascular risk. JAMA 2008;299:1265-76.

22. Agarwal A, Gupta S, Sharma RK. Role of oxidative stress in female reproduction. Reprod Biol Endocrinol 2005;3:28

23. Karbownik-Lewinska M, Marcinkowska M, Stepniak J, et al. TSH $\geq 2.5 \mathrm{mIU} / \mathrm{I}$ is Associated with the Increased Oxidative Damage to Membrane Lipids in Women of Childbearing Age with Normal Thyroid Tests. Horm Metab Res 2017;49:321-6.

24. Dobiásová M, Frohlich J. The plasma parameter log (TG/HDL-C) as an atherogenic index: correlation with lipoprotein particle size and esterification rate in apoB-lipoprotein-depleted plasma (FER(HDL)). Clin Biochem 2001;34:583-8.

25. Salazar MR, Carbajal HA, Espeche WG, et al. Identification of cardiometabolic risk: visceral adiposity index versus triglyceride/HDL cholesterol ratio. Am J Med 2014;127:152-7.

26. Kurabayashi $\mathrm{T}$, Mizunuma $\mathrm{H}$, Kubota $\mathrm{T}$, et al. Ovarian infertility is associated with cardiovascular disease risk factors in later life: A Japanese cross-sectional study. Maturitas 2016;83:33-9.

27. Luke B. Adverse effects of female obesity and interaction with race on reproductive potential. Fertil Steril 2017;107:868-77.

28. Pugh SJ, Schisterman EF, Browne RW, et al. Preconception maternal lipoprotein levels in relation to fecundability. Hum Reprod 2017;32:1055-63.

29. Kiranmayee D, Kavya K, Himabindu Y, et al. Correlations Between Anthropometry and Lipid Profile in Women With PCOS. J Hum Reprod Sci 2017;10:167-72.
30. Palomba S, Falbo A, Chiossi G, et al. Lipid profile in nonobese pregnant women with polycystic ovary syndrome: a prospective controlled clinical study. Steroids 2014;88:36-43.

31. van der Steeg JW, Steures P, Eijkemans MJ, et al. Obesity affects spontaneous pregnancy chances in subfertile, ovulatory women. Hum Reprod 2008;23:324-8.

32. Liu LX, Arany Z. Maternal cardiac metabolism in pregnancy. Cardiovasc Res 2014;101:545-53.

33. Primeau V, Coderre L, Karelis AD, et al. Characterizing the profile of obese patients who are metabolically healthy. Int $J$ Obes 2011;35:971-81.

34. Andersson G, Rønsen M, Knudsen LB, et al. Cohort Fertility Patterns in the Nordic Countries. Demogr Res 2009;20:313-52.

35. Halland F, Morken NH, DeRoo LA, et al. Association of Women's Reproductive History With Long-term Mortality and Effect of Socioeconomic Factors. Obstet Gynecol 2015;126:1181-7.

36. Spence NJ, Eberstein IW. Age at first birth, parity, and postreproductive mortality among white and black women in the US, 1982-2002. Soc Sci Med 2009;68:1625-32.

37. Magnus MC, lliodromiti S, Lawlor DA, et al. Number of Offspring and Cardiovascular Disease Risk in Men and Women: The Role of Shared Lifestyle Characteristics. Epidemiology 2017;28:880-8.

38. Craig SR, Amin RV, Russell DW, et al. Blood cholesterol screening influence of fasting state on cholesterol results and management decisions. J Gen Intern Med 2000;15:395-9.

39. Egeland GM, Klungsøyr K, Øyen N, et al. Preconception Cardiovascular Risk Factor Differences Between Gestational Hypertension and Preeclampsia: Cohort Norway Study. Hypertension 2016;67:1173-80.

40. Nordestgaard BG, Benn M, Schnohr P, et al. Nonfasting triglycerides and risk of myocardial infarction, ischemic heart disease, and death in men and women. JAMA 2007;298:299-308.

41. Sjaarda LA, Radin RG, Swanson C, et al. Prevalence and Contributors to Low-grade Inflammation in Three U.S. Populations of Reproductive Age Women. Paediatr Perinat Epidemiol 2018;32:55-67.

42. Baird DD, Wilcox AJ. Cigarette smoking associated with delayed conception. JAMA 1985;253:2979-83.

43. Maeda K, Noguchi Y, Fukui T. The effects of cessation from cigarette smoking on the lipid and lipoprotein profiles: a meta-analysis. Prev Med 2003;37:283-90.

44. Cardozo E, Pavone ME, Hirshfeld-Cytron JE. Metabolic syndrome and oocyte quality. Trends Endocrinol Metab 2011;22:103-9. 